\title{
Clues on the Galactic evolution of sulphur from star clusters ${ }^{\star}$
}

\author{
E. Caffau ${ }^{1,2 \star \star}$, L. Monaco ${ }^{3}$, M. Spite ${ }^{1}$, P. Bonifacio ${ }^{1}$, G. Carraro ${ }^{3}$, H.-G. Ludwig ${ }^{2,1}$, S. Villanova ${ }^{4}$, \\ Y. Beletsky ${ }^{5}$, and L. Sbordone $e^{6,7,2}$
}

\author{
${ }^{1}$ GEPI, Observatoire de Paris, CNRS, Univ. Paris Diderot, place Jules Janssen, 92195 Meudon, France \\ e-mail: Elisabetta.Caffau@obspm.fr \\ 2 Zentrum für Astronomie der Universität Heidelberg, Landessternwarte, Königstuhl 12, 69117 Heidelberg, Germany \\ 3 European Southern Observatory, Casilla 19001, Santiago, Chile \\ ${ }^{4}$ Departamento de Astronomía, Casilla 160, Universidad de Concepcíon, Chile \\ 5 Las Campanas Observatory, Carnegie Institution of Washington, Colina el Pino, Casilla 601, La Serena, Chile \\ ${ }^{6}$ Millennium Institute of Astrophysics, Av. Vicuña Mackenna 4860, 782-0436 Macul, Santiago, Chile \\ 7 Pontificia Universidad Católica de Chile, Av. Vicuña Mackenna 4860, 782-0436 Macul, Santiago, Chile
}

Received 24 March 2014 / Accepted 17 June 2014

\begin{abstract}
Context. The abundances of $\alpha$-elements are a powerful diagnostic of the star formation history and chemical evolution of a galaxy. Sulphur, being moderately volatile, can be reliably measured in the interstellar medium (ISM) of damped Ly- $\alpha$ galaxies and extragalactic H II regions. Measurements in stars of different metallicity in our Galaxy can then be readily compared to the abundances in external galaxies. Such a comparison is not possible for $\mathrm{Si}$ or $\mathrm{Ca}$ that suffer depletion onto dust in the ISM. Furthermore, studying sulphur is interesting because it probes nucleosynthetic conditions that are very different from those of $\mathrm{O}$ or $\mathrm{Mg}$. In this context measurements in star clusters are a reliable tracers of the Galactic evolution of sulphur.

Aims. The aim of this paper is to determine sulphur abundances in several Galactic clusters that span a metallicity range $-1.5<$ $[\mathrm{Fe} / \mathrm{H}]<0.0$.

Methods. We use a standard abundance analysis, based on 1D model atmospheres in local thermodynamical equilibrium (LTE) and literature corrections for non-LTE (NLTE), as well as 3D corrections based on hydrodynamical model atmospheres, to derive sulphur abundances in a sample of stars in the globular cluster M 4, and the open clusters Trumpler 5, NGC 2477, and NGC 5822.

Results. We find $\langle A(\mathrm{~S})\rangle_{\mathrm{NLTE}}=6.11 \pm 0.04$ for M4, $\langle A(\mathrm{~S})\rangle_{\mathrm{NLTE}}=7.17 \pm 0.02$ for NGC 2477 , and $\langle A(\mathrm{~S})\rangle_{\mathrm{NLTE}}=7.13 \pm 0.06$ for NGC 5822. For the only star studied in Trumpler 5 we find $A(\mathrm{~S})_{\text {NLTE }}=6.43 \pm 0.03$ and $A(\mathrm{~S})_{\text {LTE }}=6.94 \pm 0.05$.

Conclusions. Our measurements show that, by and large, the $\mathrm{S}$ abundances in Galactic clusters trace reliably those in field stars. The only possible exception is Trumpler 5 , for which the NLTE sulphur abundance implies an $[\mathrm{S} / \mathrm{Fe}]$ ratio lower by roughly 0.4 dex than observed in field stars of comparable metallicity, even though its LTE sulphur abundance is in line with abundances of field stars. Moreover the LTE sulphur abundance is consistent only with the abundance of another $\alpha$-element, $\mathrm{Mg}$, in the same star, while the low NLTE value is consistent with Si and Ca. We believe that further investigation of departures from LTE is necessary, as well as observation of other S I lines in this star and in other stars of the same cluster, before one can conclude that the sulphur abundance in Trumpler 5 is indeed 0.4 dex lower than in field stars of comparable metallicity. The $\mathrm{S}$ abundances in our sample of stars in clusters imply that the clusters are chemically homogeneous for $\mathrm{S}$ within 0.05 dex.
\end{abstract}

Key words. stars: abundances - Galaxy: abundances - globular clusters: individual: M 4 - Galaxy: halo

\section{Introduction}

Like $\mathrm{O}, \mathrm{Ne}, \mathrm{Mg}, \mathrm{Si}, \mathrm{Ar}, \mathrm{Ca}$, and $\mathrm{Ti}$ (the light even-elements), sulphur is an $\alpha$-element, meaning it is produced in nuclear reactions involving helium nuclei ( $\alpha$ particle). These reactions take place mainly in massive stars and, as they explode as supernovae ( $\mathrm{SNe}$ ) of Type II at the end of their life, they eject the material in the interstellar medium (ISM). The elements of the iron peak are produced by Type II $\mathrm{SNe}$ as well, and are also produced in copious amounts in Type Ia $\mathrm{SNe}$, which produce a smaller amount of $\alpha$-elements and a larger amount of iron. Different places in the Universe may have experienced different supernovae patterns and the relative measurements of the contents of $\alpha$-elements relative to the total metallicity can be very useful to learn about the past history of the stellar population

\footnotetext{
* Based on observations made with ESO Telescopes at the La Silla Paranal Observatory under programme ID 085.D-0537(A), 088.D0045(A), 089.D-0062(B).

$\star \star$ MERAC fellow.
}

and ultimately about the Universe. It could happen that one type of supernovae explodes more often than the other one, in the sense that a star ending in a Type II supernovae explosion has a much shorter life with respect to the time necessary for a star in a binary system to transfer material on its white dwarf companion and to trigger the Type Ia supernovae event; the time from the formation of the binary system to the explosion is $34 \mathrm{Myr}$ (Kobayashi \& Nomoto 2009). To study the chemical evolution of the galaxies, the timing and fraction of Type II and Type Ia $\mathrm{SNe}$, the ratio of $\alpha$-elements to iron in stars with various metallicity has to be derived. Ultimately, one can consider this ratio as an indirect measure of the star formation rate (Matteucci $\&$ Brocato 1990; Matteucci 2012). While all $\alpha$-elements are formed in the same nuclear process it should be kept in mind that this does not take place at the same location and evolutionary phase. All the $\alpha$-elements are partly formed during the star's pre-supernova evolution and partly during the explosive nucleosynthesis, except for $\mathrm{Ti}$ (which is only formed in explosive nucleosynthesis) and $\mathrm{O}$ (which is only formed in the 
pre-supernova evolution). In the pre-supernova evolution $\mathrm{O}$ is produced both in central He burning and He-shell burning; $\mathrm{Mg}$ and $\mathrm{Ne}$ are produced in the $\mathrm{C}$-convective shell burning; and $\mathrm{Si}$, $\mathrm{S}, \mathrm{Ar}$, and $\mathrm{Ca}$ are formed in the $\mathrm{O}$ convective shell burning (see Limongi \& Chieffi 2003, on-line material pages 9 and 12) ${ }^{1}$. In the explosive nucleosynthesis $\mathrm{Ne}$ is produced in the explosive carbon burning; $\mathrm{Mg}$ both in carbon and neon burning; while $\mathrm{Si}$, $\mathrm{S}, \mathrm{Ar}$, and $\mathrm{Ca}$ are produced in explosive oxygen burning and $\mathrm{Ti}$ is only produced in complete silicon burning (Limongi \& Chieffi 2003). Since all these processes operate at different temperatures and timescales there is no a priori reason why in the chemical evolution $\mathrm{O}, \mathrm{Mg}$, and $\mathrm{Si}$ (or $\mathrm{S}$, or $\mathrm{Ca}$ ) should vary in lockstep. There are instead strong reasons why $\mathrm{Si}, \mathrm{S}$, and $\mathrm{Ca}$ should vary in lockstep, since they are all produced in the same sites.

There are two reasons for the interest in studying sulphur abundances in stars. First, it probes the $\alpha$-elements that are formed in O-convective shell burning and in explosive $\mathrm{O}$ burning. Second, $\mathrm{S}$ is moderately volatile and so its abundance measured in stars in our Galaxy or Local Group galaxies can be readily compared to the abundance measured in damped Ly- $\alpha$ galaxies or extragalactic H II regions, that probe the galaxy's ISM. In fact, Si and Ca tend to be partly locked into dust in the ISM, thus the measured gas-phase abundances must be corrected for dust depletion, and such corrections are usually quite uncertain. Sulphur does not suffer from the same problem.

Although the first photospheric abundance of sulphur dates back half a century (Wallerstein \& Conti 1964), the systematic study of this element started more recently (Clegg et al. 1981; François 1987, 1988). Very few $\mathrm{S}$ indicators are present in stellar spectra. The lines of Mult. 6 and 8 at $870 \mathrm{~nm}$ and $675 \mathrm{~nm}$, respectively, are useful for solar metallicity or slightly metal-poor stars. The lines of Mult. 1 and 3 at $920 \mathrm{~nm}$ and $1045 \mathrm{~nm}$, respectively, are strong and $\mathrm{S}$ abundance can also be investigated at metalpoor regime. A forbidden line [SI] at $1082 \mathrm{~nm}$ is very useful for solar-metallicity dwarf stars, and for giant stars the metal-poor domain has also been analysed by several investigations (Caffau \& Ludwig 2007; Jönsson et al. 2011; Matrozis et al. 2013).

Most of the efforts in the $\mathrm{S}$ investigation have been dedicated to the study of the chemical evolution of sulphur in the Milky Way. The work on the $\mathrm{S}$ analysis on the Galactic field stars has been quite intense in recent years (Chen et al. 2001, 2002, 2003; Ecuvillon et al. 2004; Nissen et al. 2004; Takeda et al. 2005; Caffau et al. 2005a, 2010; Takeda \& Takada-Hidai $2011,2012)$. For an overview on the subject one can refer to the recent paper of Matrozis et al. (2013, and references therein). Spite et al. (2011) analysed S abundances in the First Stars sample of Galactic halo stars (Cayrel et al. 2004; Bonifacio et al. 2009). The most metal-poor stars $([\mathrm{Fe} / \mathrm{H}]<-2.9)$ in their sample show a mean enhancement of $[\mathrm{S} / \mathrm{Fe}]=0.35+/-0.10$, as expected in metal-poor Galactic stars. Nissen et al. (2007) analysed the Mult. 1 in a sample of metal-poor stars $(-3.3<[\mathrm{Fe} / \mathrm{H}]<$ $-1.0)$ and found an enhancement of $[\mathrm{S} / \mathrm{Fe}] \sim(+0.2 \pm 0.07)$ dex. Matrozis et al. (2013) investigate the sulphur abundance in a sample of giant stars in the range of metallicity $-2.6 \leq[\mathrm{Fe} / \mathrm{H}] \leq$ 0 by analysing the [SI] line at $1082 \mathrm{~nm}$, and found a mean enhancement of $[\mathrm{S} / \mathrm{Fe}]=+0.35$, which they argue is consistent with the enhancement of other $\alpha$-elements in metal-poor Galactic stars and with models of Galactic chemical evolution.

Less effort in the latest years has been dedicated to study $A(\mathrm{~S})$ in stars outside the Galaxy. Caffau et al. (2005b) derived $\mathrm{S}$ abundance in a sample of stars in the globular cluster (GC)

\footnotetext{
1 http://sait.oats.inaf.it/MSAIS/3/POST/Limongi_talk. $\operatorname{pdf}$
}

Terzan 7, belonging to the Sagittarius dwarf galaxy, and found a $1 \mathrm{D}$-LTE value of $\langle[\mathrm{S} / \mathrm{Fe}]\rangle=-0.05$, lower than the value derived in Galactic stars of similar iron abundance. Applying the NLTE correction from Takeda et al. (2005) the value would change by -0.12 dex.

There are only a handful of measured sulphur abundances in star clusters. Sbordone et al. (2009), adopting a Solar $A(\mathrm{~S})=7.21$, analysed two globular clusters: NGC 6752 deriving $\langle[\mathrm{S} / \mathrm{Fe}]\rangle=0.49 \pm 0.15$ consistent with the results of field stars, and 47 Tuc $\langle[\mathrm{S} / \mathrm{Fe}]\rangle=0.18 \pm 0.14$. Koch \& Caffau (2011) analysed one star in the metal-poor $([\mathrm{Fe} / \mathrm{H}]=-2.1)$ globular cluster NGC 6397 and derived $[\mathrm{S} / \mathrm{Fe}]=0.52 \pm 0.20$, with a Solar $A(\mathrm{~S})=7.14$ from Lodders et al. (2009), consistent with the value in Galactic field stars.

There are several reasons why we believe it is important to study abundances in clusters. The first and most obvious one is in order to compare the chemical evolution as traced by clusters with that traced by field stars. This helps to understand the interplay between field stars and clusters. The second is that, as clearly pointed out by Matrozis et al. (2013), the uncertainties in sulphur determinations are largely due to the uncertainty in atmospheric parameters. The study of cluster stars helps to place constraints on the atmospheric parameters from the position of the star in the HR diagram. Furthermore, on the assumption that the cluster is chemically homogeneous, one can lower the error on the sulphur abundance by averaging the abundances in different stars, if the errors in atmospheric parameters are statistical rather than systematic. The third reason is to check the abovementioned chemical homogeneity. While open clusters are still believed to be highly chemically homogeneous, globular clusters are known to show variations in the abundances of the light elements (Gratton et al. 2001, 2004). Sulphur is generally found to be homogeneous, although in 47 Tuc Sbordone et al. (2009) noticed a statistically significant correlation of $\mathrm{S}$ abundance with $\mathrm{Na}$, which is known to vary significantly in that cluster.

In this paper we provide abundances for three open and one globular cluster that span the range -1.5 to 0.0 in metallicity and attempt to use clusters as tracers of the Galactic chemical evolution of $\mathrm{S}$, using these measurements and those available in the literature.

\section{Observations and data reduction}

In this contribution, we present data obtained for stars in three open clusters (NGC 2477, NGC 5822, and Trumpler 5) and the globular cluster M 4 .

Data were obtained with the high resolution spectrograph UVES mounted at the ESO/VLT observatory, for three M 4 stars (37934, 44606, and 58 195). One 3000s exposure was taken for each star. The instrument red-arm setup analysed here covers the wavelength range $\sim 570-946 \mathrm{~nm}$. We employed a $1^{\prime \prime}$ slit width, corresponding to a resolution of $R \simeq 40000$. Data were obtained in service mode during the nights of August 19, 2012, and September 4 and 5, 2012. Data reduction was performed using the UVES CPL based pipeline version 5.3.02 . The reduced spectra have signal-to-noise ratio $(\mathrm{S} / \mathrm{N})$ in the range $25-40$ for the three stars at $\sim 800 \mathrm{~nm}$.

Data for seven stars in M 4 and for stars in NGC 2477 and NGC 5822 were obtained using the red arm of UVES, fed by the fibre-link of the multi-object facility FLAMES/VLT which provides a resolution of $R \simeq 47000$. FLAMES-UVES data was reduced using the CPL pipeline version 5.3.0. For each cluster, one

\footnotetext{
2 http://www.eso.org/sci/software/pipelines/
} 
plate configuration only was employed. One or two fibres were placed on empty regions and devoted to sky-subtraction. Spectra were cross-correlated with synthetic spectra from the Coelho et al. (2005) collection with appropriate atmospheric parameters and reported to rest frame. Finally, multiple spectra of the same star taken at different times were median combined together. FLAMES-UVES data cover the $\sim 480-680 \mathrm{~nm}$ wavelength range for the stars in NGC 2477 and NGC 5822 and $\sim 675-1000 \mathrm{~nm}$ for M 4 stars.

FLAMES-UVES M 4 data were obtained in service mode between April and July 2010 (Monaco et al. 2012, for details of the observations). Fifteen frames exposed during $2775 \mathrm{~s}$ were collected. The final spectra have $\mathrm{S} / \mathrm{N}$ in the range 35-55 at $890 \mathrm{~nm}$. Target stars were selected among turn-off and early subgiant branch stars. The photometry presented in Monaco et al. (2012) was used for the target selection. Effective temperatures, surface gravities, and microturbulence were obtained by interpolating the star's $V$ magnitudes with the ( $V$ vs. $\left.T_{\text {eff }}\right),(V$ vs. $\log g$ ), and ( $V$ vs. $\xi$ ) relations defined by the stars observed in Monaco et al. (2012). The stars analysed here cover the same CMD region as the stars analysed in Monaco et al. (2012). The adopted parameters are thus in the same scale as the Monaco et al. (2012) calibrations. In Monaco et al. (2012) all the photometric temperatures were cross-checked with the $\mathrm{H} \alpha$ temperatures obtaining a very good agreement. This is not possible for the present sample of stars because $\mathrm{H} \alpha$ is not in the wavelength range of the spectra. For the unevolved stars we adopted the same error of $150 \mathrm{~K}$ in $T_{\text {eff }}$ as in Monaco et al. (2012). We adopt, instead, an error in $T_{\text {eff }}$ of $250 \mathrm{~K}$, for the brightest stars in the sample, which approach the beginning of the subgiant branch. In this region, in fact, we have a steeper slope in the adopted $V-T_{\text {eff }}$ relation. For all the stars we adopt $0.5 \mathrm{dex}$ and $0.2 \mathrm{~km} \mathrm{~s}^{-1}$ uncertainty for the surface gravity and the micro-turbulent velocity, that are conservative estimates.

Three frames each were observed for NGC 2477 and NGC 5822 with exposure times of $1500 \mathrm{~s}$ and $1000 \mathrm{~s}$, respectively. Observations were taken during the night of October 28 and March 8, 2011, for NGC 2477 and on March 2, 7, and 25, 2011, for NGC 5822. Reduced spectra have $\mathrm{S} / \mathrm{N}$ in the range 60-90 and 90-100 at $607 \mathrm{~nm}$ for NGC 2477 and NGC 5822 stars, respectively. Targets were selected among red-clump stars using the $V$ vs. $B-V$ photometry of Kassis et al. (1997) and Carraro et al. (2011) for the two clusters. To derive the stellar parameters of both clusters we analysed the spectra with the code MyGIsFOS (Sbordone et al. 2014). The effective temperature is derived by requiring no trend in the iron abundance as a function of the lower energy of the Fe I lines; the gravity by matching the iron abundance derived from Fe I and Fe II lines; the microturbulence by requiring no slope in the iron abundance as a function of the equivalent width of the Fe I lines. In this way we derive the iron abundance for each star. The ratio $[\mathrm{S} / \mathrm{Fe}]$ is less dependent on the stellar parameters than either the abundance of sulphur and iron, since the errors tend to cancel out when considering the abundance ratios.

Observations of one red-clump star in the open cluster Trumpler 5 was obtained with the high resolution spectrograph MIKE mounted at the Magellan-II/Clay telescope. Five $1800 \mathrm{~s}$ frames were taken on October 19,2013. We used a 0.7 arcsec slit, providing a resolution $R \simeq 42000$. The spectra were reduced using the MIKE pipeline ${ }^{3}$ and cover the wavelength range $\sim 353-940 \mathrm{~nm}$. The median spectrum has a $\mathrm{S} / \mathrm{N}$ of about $20-30$ at $920 \mathrm{~nm}$. Atmospheric parameters for this star were derived from

\footnotetext{
3 http://web.mit.edu/ burles/www/MIKE/
}

Table 1. Transitions and $\log g f$ of Mult. 8 and Mult. 1 from Wiese et al. (1969).

\begin{tabular}{ccccc}
\hline \hline & Transition & $\begin{array}{c}\lambda \\
(\mathrm{nm})\end{array}$ & $\log g f$ & $\begin{array}{c}E_{\text {low }} \\
(\mathrm{eV})\end{array}$ \\
\hline Mult. 8 & & & & \\
$4 \mathrm{p}-5 \mathrm{~d}$ & ${ }^{5} \mathrm{P}_{1}-{ }^{5} \mathrm{D}^{\circ}{ }_{0}$ & 674.3440 & -1.27 & 7.866 \\
& ${ }^{5} \mathrm{P}_{1}-{ }^{5} \mathrm{D}^{\circ}{ }_{1}$ & 674.3531 & -0.92 & 7.866 \\
& ${ }^{5} \mathrm{P}_{1}-{ }^{5} \mathrm{D}^{\circ}{ }_{2}$ & 674.3640 & -1.03 & 7.866 \\
& ${ }^{5} \mathrm{P}_{2}-{ }^{5} \mathrm{D}^{\circ}{ }_{1}$ & 674.8573 & -1.39 & 7.868 \\
& ${ }^{5} \mathrm{P}_{2}-{ }^{5} \mathrm{D}^{\circ}{ }_{2}$ & 674.8682 & -0.80 & 7.868 \\
& ${ }^{5} \mathrm{P}_{2}-{ }^{5} \mathrm{D}^{\circ}{ }_{3}$ & 674.8837 & -0.60 & 7.868 \\
& ${ }^{5} \mathrm{P}_{3}-{ }^{5} \mathrm{D}^{\circ}{ }_{2}$ & 675.6851 & -1.76 & 7.870 \\
& ${ }^{5} \mathrm{P}_{3}-{ }^{5} \mathrm{D}^{\circ}{ }_{3}$ & 675.7007 & -0.90 & 7.870 \\
& ${ }^{5} \mathrm{P}_{3}-{ }^{5} \mathrm{D}^{\circ}{ }_{4}$ & 675.7171 & -0.31 & 7.870 \\
\hline Mult. 1 & & & & \\
$4 \mathrm{~s}-4 \mathrm{p}$ & ${ }^{5} \mathrm{~S}_{2}{ }_{2}{ }^{5} \mathrm{P}_{1}$ & 923.7538 & 0.04 & 6.525 \\
& ${ }^{5} \mathrm{~S}^{\circ}{ }_{2}-{ }^{5} \mathrm{P}_{2}$ & 922.8093 & 0.26 & 6.525 \\
& ${ }^{5} \mathrm{~S}_{2}^{\circ}{ }^{5} \mathrm{P}_{3}$ & 921.2863 & 0.42 & 6.525 \\
\hline
\end{tabular}

a detailed chemical abundance analysis similar to the other two open clusters, but in this case the code MyGIsFOS was not used and the analysis was done in the traditional way. In the analysis of this star, we used the photometry and cluster parameters from Kaluzny (1998; see Monaco et al. 2014, for details) .

\section{Analysis}

We analysed the $\mathrm{S}$ I lines of the Multiplet 1 at $920 \mathrm{~nm}$ in a sample of stars in M4 and a star in Trumpler 5 and Mult. 8 in a sample of stars in two open clusters, NGC 2477 and NGC 5822. The S I lines of Mult. 6 and Mult. 8 are also present in the wavelength ranges covered by our spectra of M 4, but the lines are too weak to be detected. Atomic parameters of the multiplets are shown in Table 1.

The spectral region of Mult. 1 is known to be contaminated by telluric absorption. We compared each observed spectrum with a synthetic profile that reconstructs the absorption of the terrestrial atmosphere at the site and time of the observation by using TAPAS ${ }^{4}$. An example is shown in Fig. 1 . The S I lines in the sample of M 4 are mostly clean from telluric absorption for all stars and we analysed all S I lines in the complete sample of stars. For the star M4-37934, the reddest S I line seems to be contaminated by a weak telluric absorption line (see Fig. 1), but we consider this contribution minor, and kept this line in the analysis. Discarding it would reduce $A(\mathrm{~S})$ by 0.02 dex. For star 3416 in Trumpler 5 the reddest line is strongly contaminated by a telluric absorption which means that the line is not useful for abundance analysis. In addition the line at $922.8 \mathrm{~nm}$ has a tiny telluric component (see Fig. 2) that we consider negligible.

We computed an ATLAS 9 model (Kurucz 1993a, 2005) for each star of M4, with the parameters reported in Table 2. We computed synthetic profiles with the code SYNTHE (Kurucz 1993b, 2005) in its Linux version (Sbordone et al. 2004; Sbordone 2005), with different abundances of sulphur. We derived $A(\mathrm{~S})$ fitting each line, minimising the $\chi^{2}$, with the code described in Bonifacio \& Caffau (2003). As solar sulphur abundance we take $A(\mathrm{~S})=7.16$ and for iron $A(\mathrm{Fe})=7.52$ (Caffau et al. 2011). The $\mathrm{S}$ abundances we derived are presented

4 http://ether.ipsl.jussieu.fr/tapas/(Bertaux et al. 2012). 
Table 2. Stellar parameters and S abundances.

\begin{tabular}{|c|c|c|c|c|c|c|c|}
\hline Star & $\begin{array}{l}T_{\text {eff }} \\
\mathrm{K} \\
\end{array}$ & $\begin{array}{c}\log g \\
\text { [c.g.s.] }\end{array}$ & $\begin{array}{r}{[\mathrm{M} / \mathrm{H}]^{a}} \\
\operatorname{dex} \\
\end{array}$ & $\begin{array}{l}\xi \\
\mathrm{km} \mathrm{s}^{-1}\end{array}$ & $\begin{array}{r}V_{\mathrm{r}} \\
\mathrm{km} \mathrm{s}^{-1}\end{array}$ & $\begin{array}{c}A(\mathrm{~S}) \\
\text { 1D-LTE }\end{array}$ & $\begin{array}{c}A(\mathrm{~S}) \\
\text { 1D-NLTE }\end{array}$ \\
\hline \multicolumn{8}{|l|}{ M4 } \\
\hline $\begin{array}{l}8042 \\
30303 \\
31746 \\
34380 \\
37934 \\
44606 \\
48721 \\
58195 \\
64845 \\
67935 \\
\end{array}$ & $\begin{array}{l}5965 \\
5812 \\
5887 \\
5899 \\
5979 \\
5966 \\
5819 \\
5972 \\
5828 \\
5923 \\
\end{array}$ & $\begin{array}{l}4.05 \\
3.87 \\
3.94 \\
3.96 \\
4.11 \\
4.06 \\
3.88 \\
4.08 \\
3.89 \\
3.98 \\
\end{array}$ & $\begin{array}{l}-1.36 \\
-1.36 \\
-1.36 \\
-1.36 \\
-1.36 \\
-1.36 \\
-1.36 \\
-1.36 \\
-1.36 \\
-1.36 \\
\end{array}$ & $\begin{array}{l}1.7 \\
1.7 \\
1.7 \\
1.7 \\
1.7 \\
1.7 \\
1.7 \\
1.7 \\
1.7 \\
1.7 \\
\end{array}$ & $\begin{array}{l}62.45 \pm 1.15 \\
70.01 \pm 2.17 \\
63.55 \pm 1.07 \\
74.00 \pm 1.08 \\
66.44 \pm 0.30 \\
74.54 \pm 1.34 \\
68.12 \pm 1.19 \\
67.98 \pm 0.37 \\
73.14 \pm 1.13 \\
66.46 \pm 1.22 \\
\end{array}$ & $\begin{array}{l}6.32 \pm 0.09 \\
6.39 \pm 0.10 \\
6.39 \pm 0.15 \\
6.35 \pm 0.12 \\
6.38 \pm 0.08 \\
6.28 \pm 0.13 \\
6.35 \pm 0.09 \\
6.25 \pm 0.07 \\
6.32 \pm 0.09 \\
6.28 \pm 0.18 \\
\end{array}$ & $\begin{array}{l}6.12 \pm 0.05 \\
6.08 \pm 0.05 \\
6.15 \pm 0.07 \\
6.13 \pm 0.03 \\
6.18 \pm 0.09 \\
6.09 \pm 0.12 \\
6.12 \pm 0.04 \\
6.04 \pm 0.05 \\
6.10 \pm 0.04 \\
6.08 \pm 0.13\end{array}$ \\
\hline \multicolumn{8}{|l|}{$\operatorname{Tr} 5$} \\
\hline 3416 & 4870 & 2.05 & -0.53 & 1.33 & $50.5 \pm 0.2$ & $6.94 \pm 0.05$ & $6.43 \pm 0.03$ \\
\hline \multicolumn{8}{|c|}{ NGC 5822} \\
\hline $\begin{array}{l}2397 \\
13292 \\
16450 \\
18897 \\
\end{array}$ & $\begin{array}{l}5145 \\
5066 \\
5017 \\
5115 \\
\end{array}$ & $\begin{array}{l}2.95 \\
2.80 \\
2.71 \\
3.00 \\
\end{array}$ & $\begin{array}{r}0.05 \\
0.05 \\
-0.01 \\
-0.02 \\
\end{array}$ & $\begin{array}{l}1.13 \\
1.12 \\
1.09 \\
1.10 \\
\end{array}$ & $\begin{array}{l}-29.67 \pm 0.79 \\
-29.35 \pm 0.34 \\
-25.69 \pm 0.37 \\
-29.01 \pm 0.22 \\
\end{array}$ & $\begin{array}{l}7.11 \pm 0.02 \\
7.16 \pm 0.04 \\
7.17 \pm 0.01 \\
7.09 \pm 0.02 \\
\end{array}$ & \\
\hline \multicolumn{8}{|c|}{ NGC 2477} \\
\hline $\begin{array}{l}4027 \\
4221 \\
5043 \\
5076 \\
7266 \\
7273 \\
8216 \\
\end{array}$ & $\begin{array}{l}4998 \\
4956 \\
5075 \\
5010 \\
5036 \\
4977 \\
5017 \\
\end{array}$ & $\begin{array}{l}2.78 \\
2.70 \\
2.96 \\
2.80 \\
2.92 \\
2.67 \\
2.84 \\
\end{array}$ & $\begin{array}{l}0.14 \\
0.08 \\
0.04 \\
0.07 \\
0.13 \\
0.02 \\
0.12 \\
\end{array}$ & $\begin{array}{l}1.12 \\
1.12 \\
1.06 \\
1.14 \\
1.09 \\
1.20 \\
0.99 \\
\end{array}$ & $\begin{array}{r}7.03 \pm 0.13 \\
8.80 \pm 0.23 \\
13.22 \pm 0.28 \\
9.22 \pm 0.33 \\
9.30 \pm 0.14 \\
8.77 \pm 0.51 \\
3.99 \pm 0.50\end{array}$ & $\begin{array}{l}7.30 \pm 0.06 \\
7.28 \pm 0.04 \\
7.18 \pm 0.06 \\
7.20 \pm 0.06 \\
7.27 \pm 0.08 \\
7.22 \pm 0.01 \\
7.24 \pm 0.05\end{array}$ & \\
\hline
\end{tabular}

Notes. The uncertainty is the line-to-line scatter. ${ }^{(a)} 10^{[\mathrm{M} / \mathrm{H}]}$ is the scaling factor applied to the abundances in the model, in the $[\mathrm{M} / \mathrm{H}]=-1.5$ models, the $\alpha$-elements are enhanced by 0.4 dex.

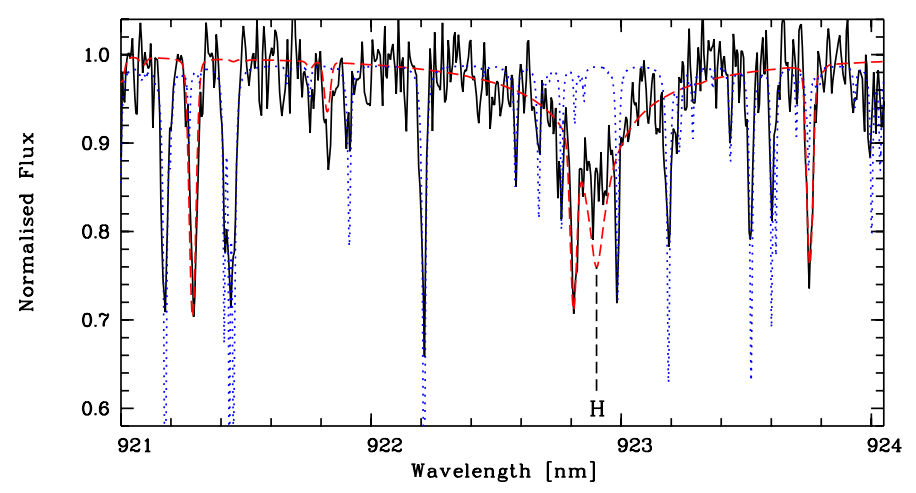

Fig. 1. Observed profile of M 4-37934 (solid black) superimposed on the synthetic profile with the derived average $A(\mathrm{~S})$ (dashed red) and the synthetic telluric profile derived from Tapas (dotted blue). The hydrogen line Paschen $\zeta$ is indicated in the plot.

in Table 2. The line-to-line scatter is on average $0.11 \mathrm{dex}$ for the 1D-LTE abundances and decreases to an average value of 0.07 dex for the 1D-NLTE value. The same approach was used for the star of Trumpler 5 .

In the same way, we also analysed the lines of Mult. 8 in the stars of two open clusters, NGC 2477 and NGC 5822 (see Table 2). An example of the line profile fitting is shown in Fig. 3. To compute the line-to-line scatter we gave double weight to the reddest $\mathrm{S}$ I feature at $675.7 \mathrm{~nm}$ because it is the strongest.

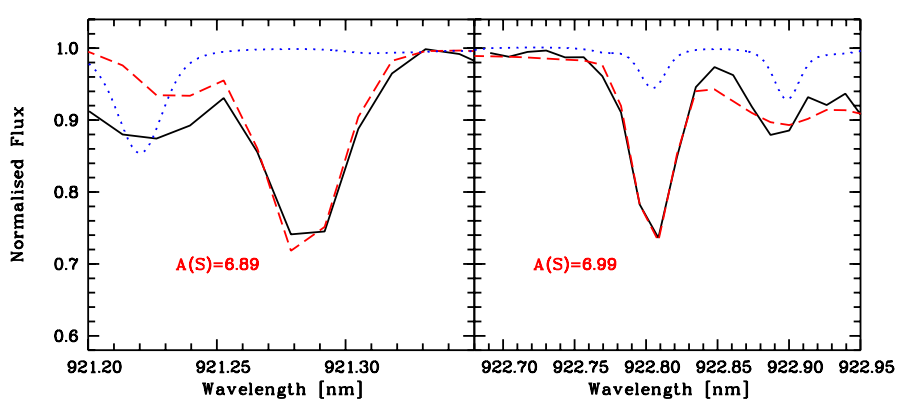

Fig. 2. Observed spectrum of the star 3416 in Trumpler 5 (solid black) superimposed on the best fit (dashed red). The contribution to the spectrum due to the terrestrial atmosphere is also shown (dotted blue).

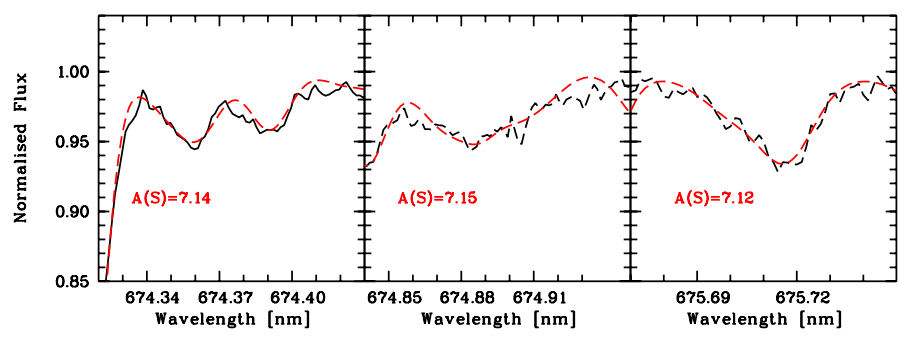

Fig. 3. The three S I lines in the observed profile of star 16450 in NGC 5822 (solid black) superimposed on the synthetic profile with the derived $A(\mathrm{~S})$ (dashed red).

We obtained on average a line-to-line scatter of 0.02 dex and 0.05 dex for NGC 2477 and NGC 5822, respectively. 
E. Caffau et al.: Sulphur in star clusters

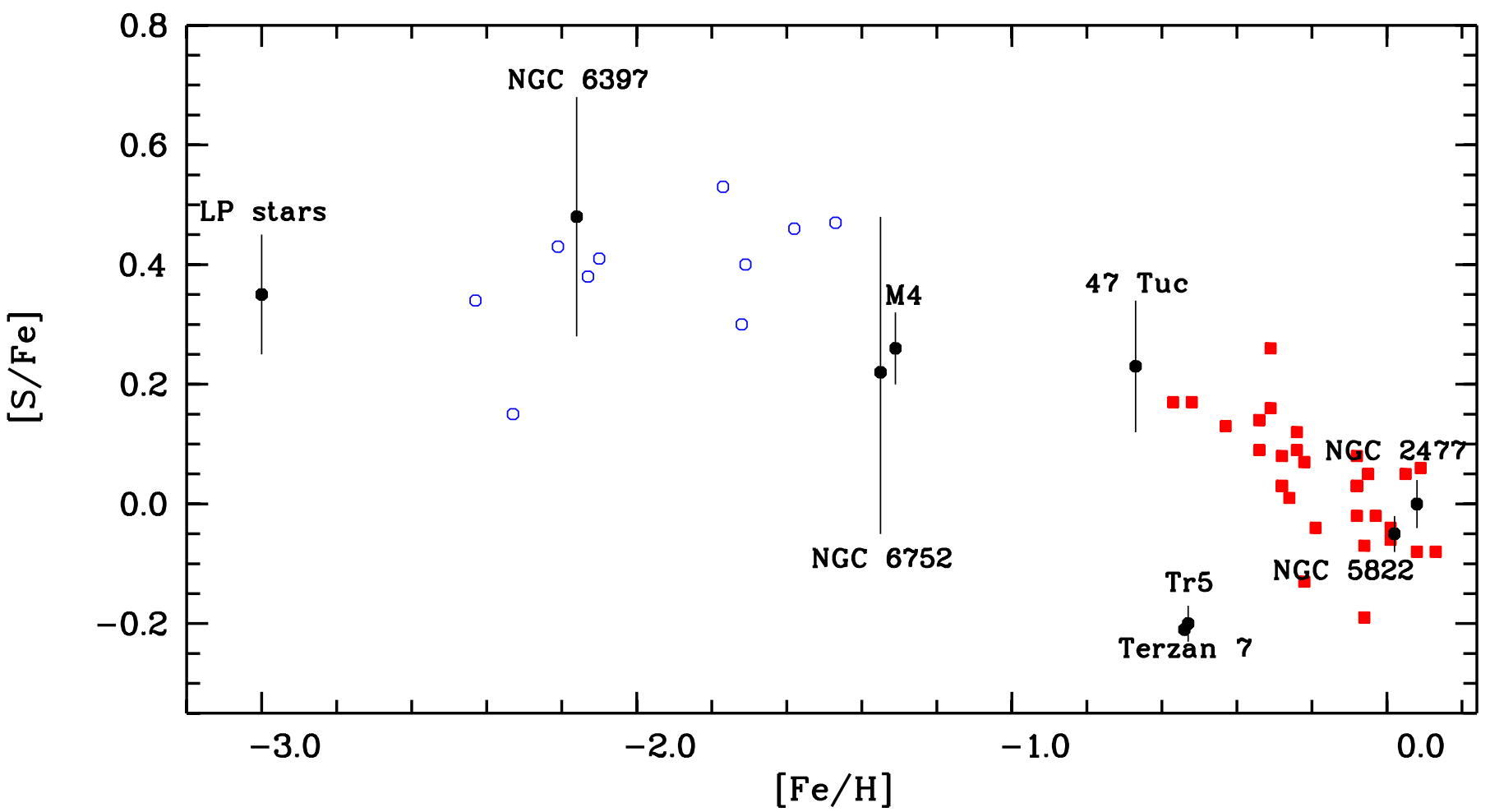

Fig. 4. [S/Fe] vs. iron content for the clusters with known S abundance. NGC 6397 from Koch \& Caffau (2011); NGC 6752 and 47 Tuc from Sbordone et al. (2009); Terzan 7 from Caffau et al. (2005b). The results of M 4 and Trumpler 5, based on Mult. 1, are discussed in this paper. The analysis of NGC 5822 and NGC 2477 are based on the lines of Mult. 8. For comparison we plot sulphur abundances in Galactic stars. "LP stars" is an average in metallicity and $[\mathrm{S} / \mathrm{Fe}]$ for the stars with $[\mathrm{Fe} / \mathrm{H}]<-2.9$ from Spite et al. (2011), from Mult. 1; the metal-poor sample from Jönsson et al. (2011) (blue open circles) based on Mult. 3, and from the forbidden [SI] line at $108.2 \mathrm{~nm}$ line; the solar-metallicity sample of comparison stars from Ecuvillon et al. (2004) (red filled squares) where Mult. 8 has been analysed. All the points have been scaled to our adopted solar abundances $A(\mathrm{~S}) \odot=7.16$ and $A(\mathrm{Fe}) \odot=7.52($ Caffau et al. 2011).

We derived the NLTE corrections for the lines of Mult. 1 from Takeda et al. (2005). The 3D corrections are about +0.23 , +0.22 , and +0.20 dex, respectively, for the 1D-LTE abundance of the three lines in the stars of M 4. They were computed interpolating the two closest models of the CIFIST grid (Ludwig et al. 2009), $5900 \mathrm{~K} / 4.0 /-1.0$ and $5900 \mathrm{~K} / 4.0 /-2.0$. For details on the computations of 3D effects see Caffau \& Ludwig (2007). To take into account the $3 \mathrm{D}$ effects, we can add 0.2 dex to the values of $A(\mathrm{~S})$-NLTE we find. For the star of Trumpler 5, taking from the CIFIST grid two models with parameters $5000 \mathrm{~K} / 2.5 / 0.0$ and $5000 \mathrm{~K} / 2.5 /-1$, we derive a very similar 3D correction of +0.25 . The 3D corrections for the lines of Mult. 8 in solar metallicity stars are negligible. We verified this fact by looking at the 3D model with parameters $5000 \mathrm{~K} / 3.5 / 0.0$. The NLTE corrections for the lines of Mult. 8 were computed by Korotin (2008) and are very small $\left(-0.1\right.$ dex $\left.<\Delta_{\text {NLTE }}<0\right)$. Considering that they are smaller than our errors we did not apply them. A 1D-LTE computation by adding the NLTE corrections and the 3D corrections surely cannot substitute the 3D-NLTE computation that would be desirable, but this is what we can do at the moment. We can hope that the complete 3D-NLTE computation is close to our approach. In the case of the S I lines of Mult. 1 for these stars, they form in the stellar photosphere, around $\log _{10} \tau \approx-0.5$ both in the case of the 3D and 1D computations. For lines forming deep in the stellar photosphere as the S I lines of Mult. 1 the abundance derived could depend on the choice of the mixing length parameter. A change of the mixing length parameter from 1.25 (the default value used in ATLAS) to 0.5 implies a change in the abundance of less than $0.01 \mathrm{dex}$, a negligible value for the quality of our observations.
We investigated the impact of uncertainty of the stellar parameters on the sulphur abundance we derive. We adopt for the stars of M4 a conservative uncertainty of $150 \mathrm{~K}, 0.5 \mathrm{dex}$, and $0.2 \mathrm{~km} \mathrm{~s}^{-1}$ in effective temperature, gravity, and microturbulence. We adopt a larger uncertainty of $250 \mathrm{~K}$ in $T_{\text {eff }}$ for the five stars in the sample of M $4(30303,31746,34380,48721,64845)$, close to the SGB region. This leads to an uncertainty in $A(\mathrm{~S})$ derived from uncertainty in the stellar parameters for the lines of Mult. 1 of $0.1 \mathrm{dex}$ in the unevolved stars and $0.13 \mathrm{dex}$ in the evolved stars of M 4, and of 0.17 dex for the star of Trumpler 5 . For all the stars for which the sulphur abundance has been determined from Mult.1 the error related to the uncertainty in the microturbulence is not negligible and the uncertainties on the stellar parameters contribute in a comparable way to the systematic error on $A(\mathrm{~S})$. For the stars of the two open clusters where $A(\mathrm{~S})$ is derived from the $\mathrm{S}$ I lines of Mult. 8, with an uncertainty of $100 \mathrm{~K}$ in temperature, $0.5 \mathrm{dex}$ in gravity, and $0.2 \mathrm{~km} \mathrm{~s}^{-1}$ in microturbulence, we have instead an abundance uncertainty of $\sigma=0.19 \mathrm{dex}$, in this case the error due to an uncertainty in gravity is about twice the error related to effective temperature, while the microturbulence has a negligible effect. From the above discussion and the line-to-line scatter given in Table 2, it is clear that the total error is generally dominated by the systematic error due to the uncertainty in atmospheric parameters. This implies that our data has an adequate $\mathrm{S} / \mathrm{N}$ for this work.

\section{Discussion}

In Fig. 4, $[\mathrm{S} / \mathrm{Fe}]$ is plotted as a function of $[\mathrm{Fe} / \mathrm{H}]$ for the clusters analysed in this work and for literature data on clusters and field 
stars. The sample of stars of M4 shows a homogeneous amount of sulphur, $\langle A(\mathrm{~S})\rangle_{\mathrm{LTE}}=6.33 \pm 0.05$ and $\langle A(\mathrm{~S})\rangle_{\mathrm{NLTE}}=6.11 \pm$ 0.04. The star-to-star scatter is comparable with the line-to-line scatter and with the systematic uncertainty. The NLTE correction is about 0.2 dex, a value that does not change the general picture of the cluster. NGC 5822 and NGC 2477 both show a sulphur abundance comparable to Galactic field stars, $\langle A(\mathrm{~S})\rangle=7.13 \pm$ 0.03 and $\langle A(\mathrm{~S})\rangle=7.24 \pm 0.04$, respectively. In NGC 5822 one star, 16450 has a $V_{\text {rad }}$ somewhat in disagreement with the other three stars, but its $\mathrm{S}$ abundance is in agreement with the other stars, as expected from field stars. Removing this star from the sample would not affect the mean $A(\mathrm{~S})$. In NGC 2477 there are two outliers in radial velocity, stars 5043 and 8216. Star 5043 shows the lowest $A(\mathrm{~S})$ value, while the other is at the average. By removing 5043 from the sample we do not have a significant difference, $\sigma,\langle A(\mathrm{~S})\rangle=7.25 \pm 0.03$.

The real surprise comes from the only star observed in Trumpler 5: it seems to behave like a Sagittarius cluster star with an under-abundance of $S$ with respect to iron, when compared to the Galactic behaviour. In this case it would be similar to the cases of Berkely 29 and Saurer 1 (Carraro \& Bensby 2009). We can see that the LTE abundance would give $[\mathrm{S} / \mathrm{Fe}]=+0.3$, in agreement with the $1 \mathrm{D}-\mathrm{LTE}$ value of $[\mathrm{Mg} / \mathrm{Fe}]=+0.27$ (Monaco et al. 2014). It could seem that it is the NLTE correction for this star that drives its anomalously low S abundance; we note however that the NLTE correction computed by Korotin (2008) for this line is of about the same size. If we compare $[\mathrm{S} / \mathrm{Fe}]$ to $[\mathrm{Si} / \mathrm{Fe}]$ and $[\mathrm{Ca} / \mathrm{Fe}]$, they are both slightly lower than expected, +0.08 and +0.18 , respectively (Monaco et al. 2014). We have not performed NLTE calculations for $\mathrm{Si}, \mathrm{Ca}$, and $\mathrm{Mg}$ in this star; however by looking at published NLTE computations for $\mathrm{Mg}$ (Gehren et al. 2004; Andrievsky et al. 2010; Merle et al. 2011), Si (Shi et al. 2009), and Ca (Mashonkina et al. 2007; Spite et al. 2012) we expect the corrections to be small and positive. If this were confirmed, it could be the first evidence that $\mathrm{S}$ can vary in a slightly different way than $\mathrm{Mg}$. The situation for $\mathrm{Si}$ and $\mathrm{Ca}$ depends significantly on the magnitude and direction of the NLTE corrections.

The main conclusion of our investigation is that the Galactic clusters show the same evolution in sulphur as Galactic stars, while the cluster Terzan 7, which belongs to Sagittarius, has a lower S abundance, as expected for Sagittarius that shows low $\alpha$-to-iron ratios (Bonifacio et al. 2004; Monaco et al. 2005, 2007; Sbordone et al. 2007). We hesitate to trust the low sulphur abundance found in the Trumpler 5 star, after applying the NLTE correction, since the LTE abundance would be more coherent with that in Galactic stars and abundances of $\mathrm{Mg}$ in the same star, although $\mathrm{Si}$ and $\mathrm{Ca}$ seem to be slightly less enhanced than $\mathrm{Mg}$. Further investigation of NLTE effects on sulphur and measurement of other stars in this cluster, possibly from other lines, would clearly be useful, as well as investigation of NLTE effects on $\mathrm{Mg}, \mathrm{Si}$, and $\mathrm{Ca}$. The globular cluster $\mathrm{M} 4$, for which we could measure $S$ in ten stars, does not show any variation in $S$ abundances, suggesting that $S$ does not participate to the chemical variations found for lighter elements $(\mathrm{Li}, \mathrm{C}, \mathrm{N}, \mathrm{O}, \mathrm{Na}, \mathrm{Mg}, \mathrm{Al})$ in globular clusters (see Gratton et al. 2004, for a review). In particular for M 4 the variations of these elements were found by Ivans et al. (1999) and Monaco et al. (2012). This confirms the similar finding for NGC 6752 by Sbordone et al. (2009), that has a comparable metallicity. There is still no general consensus on what stars are responsible for the light element variations in globular clusters, although it is clear that they cannot be $\mathrm{SNe}$, since their explosions would blow out all the gas from the cluster preventing any further star formation; knowing which elements vary and which do not places a further constraint on the nature of the polluters. The case of 47 Tuc is, to date, the only GC for which a variation in $\mathrm{S}$ abundances has been suspected (Sbordone et al. 2009); it ought to be revisited, and further observations of $S$ in this cluster are encouraged. Further observations in more metalpoor globular clusters, like NGC 6397 and including NGC 6397 itself for which a single star has been measured, as well as in globular clusters around metallicity -1.0 , are badly needed to refine the picture that is emerging from the presently available observations.

Acknowledgements. The project was funded by FONDATION MERAC. E.C., H.G.L., and L.S. acknowledge financial support by the Sonderforschungsbereich SFB881 "The Milky Way System" (subprojects A4 and A5) of the German Research Foundation (DFG). P.B. and M.S. acknowledge support from the Programme National de Cosmologie et Galaxies (PNCG) of the Institut National des Sciences de l'Univers of CNRS. S.V. gratefully acknowledges the support provided by FONDECYT No. 1130721. L.S. aknowledges financial support from Project IC120009 "Millennium Institute of Astrophysics (MAS)" of Iniciativa Científica Milenio del Ministerio de Economía, Fomento y Turismo de Chile.

\section{References}

Andrievsky, S. M., Spite, M., Korotin, S. A., et al. 2010, A\&A, 509, A88

Bertaux, J. L., Lallement, R., Ferron, S., Boonne, C., \& Bodichon, R. 2012, TAPAS ASA/HITRAN conference, 2012 Reims, France

Bonifacio, P., \& Caffau, E. 2003, A\&A, 399, 1183

Bonifacio, P., Sbordone, L., Marconi, G., Pasquini, L., \& Hill, V. 2004, A\&A, 414, 503

Bonifacio, P., Spite, M., Cayrel, R., et al. 2009, A\&A, 501, 519

Caffau, E., \& Ludwig, H.-G. 2007, A\&A, 467, L11

Caffau, E., Bonifacio, P., Faraggiana, R., et al. 2005a, A\&A, 441, 533

Caffau, E., Bonifacio, P., Faraggiana, R., \& Sbordone, L. 2005b, A\&A, 436, L9

Caffau, E., Sbordone, L., Ludwig, H.-G., Bonifacio, P., \& Spite, M. 2010, Astron. Nachr., 331, 725

Caffau, E., Ludwig, H.-G., Steffen, M., Freytag, B., \& Bonifacio, P. 2011, Sol. Phys., 268, 255

Cayrel, R., Depagne, E., Spite, M., et al. 2004, A\&A, 416, 1117

Carraro, G., \& Bensby, T. 2009, MNRAS, 397, L106

Carraro, G., Anthony-Twarog, B. J., Costa, E., Jones, B. J., \& Twarog, B. A. 2011, AJ, 142, 127

Chen, Y. Q., Nissen, P. E., Benoni, T., \& Zhao, G. 2001, A\&A, 371, 943

Chen, Y. Q., Nissen, P. E., Zhao, G., \& Asplund, M. 2002, A\&A, 390, 225

Chen, Y. Q., Zhao, G., Nissen, P. E., Bai, G. S., \& Qiu, H. M. 2003, ApJ, 591, 925

Clegg, R. E. S., Tomkin, J., \& Lambert, D. L. 1981, ApJ, 250, 262

Coelho, P., Barbuy, B., Meléndez, J., Schiavon, R. P., \& Castilho, B. V. 2005, A\&A, 443, 735

Ecuvillon, A., Israelian, G., Santos, N. C., et al. 2004, A\&A, 426, 619

François, P. 1987, A\&A, 176, 294

François, P. 1988, A\&A, 195, 226

Gehren, T., Liang, Y. C., Shi, J. R., Zhang, H. W., \& Zhao, G. 2004, A\&A, 413, 1045

Gratton, R. G., Bonifacio, P., Bragaglia, A., et al. 2001, A\&A, 369, 87

Gratton, R., Sneden, C., \& Carretta, E. 2004, ARA\&A, 42, 385

Ivans, I. I., Sneden, C., Kraft, R. P., et al. 1999, AJ, 118, 1273

Jönsson, H., Ryde, N., Nissen, P. E., et al. 2011, A\&A, 530, A144

Kaluzny, J. 1998, A\&AS, 133, 25 (K98)

Kassis, M., Janes, K. A., Friel, E. D., \& Phelps, R. L. 1997, AJ, 113, 1723

Kobayashi, C., \& Nomoto, K. 2009, ApJ, 707, 1466

Koch, A., \& Caffau, E. 2011, A\&A, 534, A52

Korotin, S. A. 2008, Odessa Astron. Publ., 21, 42

Kurucz, R. 1993a, ATLAS9 Stellar Atmosphere Programs and $2 \mathrm{~km} \mathrm{~s}^{-1}$ grid. Kurucz CD-ROM No. 13 (Cambridge, MA: Smithsonian Astrophysical Observatory)

Kurucz, R. 1993b, SYNTHE Spectrum Synthesis Programs and Line Data. Kurucz CD-ROM (Cambridge, MA: Smithsonian Astrophysical Observatory), 18

Kurucz, R. L. 2005, Mem. Soc. Astron. It. Suppl., 8, 14

Limongi, M., \& Chieffi, A. 2003, Mem. Soc. Astron. It. Suppl., 3, 58

Lodders, K., Plame, H., \& Gail, H.-P. 2009, Landolt-Börnstein - Group VI Astronomy and Astrophysics Numerical Data and Functional Relationships in Science and Technology Vol. 4B: Solar System, ed. J. E. Trümper, 4.4, 44 
E. Caffau et al.: Sulphur in star clusters

Ludwig, H.-G., Caffau, E., Steffen, M., et al. 2009, Mem. Soc. Astron. It., 80, 711

Mashonkina, L., Korn, A. J., \& Przybilla, N. 2007, A\&A, 461, 261

Matrozis, E., Ryde, N., \& Dupree, A. K. 2013, A\&A, 559, A115

Matteucci, F. 2012, Chemical Evolution of Galaxies: Astronomy and Astrophysics Library (Berlin, Heidelberg: Springer-Verlag)

Matteucci, F., \& Brocato, E. 1990, ApJ, 365, 539

Merle, T., Thévenin, F., Pichon, B., \& Bigot, L. 2011, MNRAS, 418, 863

Monaco, L., Bellazzini, M., Bonifacio, P., et al. 2005, A\&A, 441, 141

Monaco, L., Bellazzini, M., Bonifacio, P., et al. 2007, A\&A, 464, 201

Monaco, L., Villanova, S., Bonifacio, P., et al. 2012, A\&A, 539, A157

Monaco, L., Boffin, H. M. J., Bonifacio, P., et al. 2014, A\&A, 564, L6

Nissen, P. E., Chen, Y. Q., Asplund, M., \& Pettini, M. 2004, A\&A, 415, 993

Nissen, P. E., Akerman, C., Asplund, M., et al. 2007, A\&A, 469, 319
Sbordone, L. 2005, Mem. Soc. Astron. It. Suppl., 8, 61

Sbordone, L., Bonifacio, P., Castelli, F., \& Kurucz, R. L. 2004, Mem. Soc. Astron. It. Suppl., 5, 93

Sbordone, L., Bonifacio, P., Buonanno, R., et al. 2007, A\&A, 465, 815

Sbordone, L., Limongi, M., Chieffi, A., et al. 2009, A\&A, 503, 121

Sbordone, L., Caffau, E., Bonifacio, P., \& Duffau, S. 2014, A\&A, 564, A109

Shi, J. R., Gehren, T., Mashonkina, L., \& Zhao, G. 2009, A\&A, 503, 533

Spite, M., Caffau, E., Andrievsky, S. M., et al. 2011, A\&A, 528, A9

Spite, M., Andrievsky, S. M., Spite, F., et al. 2012, A\&A, 541, A143

Takeda, Y., \& Takada-Hidai, M. 2011, PASJ, 63, 537

Takeda, Y., \& Takada-Hidai, M. 2012, PASJ, 64, 42

Takeda, Y., Hashimoto, O., Taguchi, H., et al. 2005, PASJ, 57, 751

Wallerstein, G., \& Conti, P. 1964, ApJ, 140, 858

Wiese, W. L., Smith, M. W., \& Miles, B. M. 1969, in NSRDS-NBS (Washington, D.C.: US Department of Commerce, National Bureau of Standards) 